\title{
Experimental Study on Generation Mechanism and Influencing Factors of Metal Machining Fluid Oil Mist Microparticles
}

\author{
Changhe Li ${ }^{* 1}$, Huayang Zhao ${ }^{2}$, Dongzhou Jia ${ }^{1}$, Dongkun Zhang ${ }^{1}$ and Yali Hou ${ }^{1}$ \\ ${ }^{1}$ School of Mechanical Engineering, Qingdao Technological University, 266033 \\ Qingdao, China \\ ${ }^{2}$ School of Mechanical Engineering, Inner Mongolia University for the Nationalities, \\ 028043 Tongliao, China \\ *sy_lichanghe@163.com
}

\begin{abstract}
Based on the dangers of suspended oil mist microparticles from machining to the environment and health of worker. A theoretical analysis on the generation mechanism of cutting fluid oil mist and an experimental study on its influencing factors were carried out in this paper. The experiment was conducted in closed lathe with flood cutting fluid feed system. A droplet diameter measuring system was equipped in the dense region of oil mist, which enables to observe the droplet distribution in air and scan the morphology of a single droplet accurately through its confocal microscopy. Droplet distribution and diameter were calculated through computer. In the experiment, effects of influencing factors on the oil mist concentration and average droplet diameter were discussed by controlling the revolutions per minute (rpm) of the main axis and cutting fluid flow, respectively. Experimental results demonstrated a significant effect of rpm on the characteristics of cutting fluid oil mist. As the rpm increased, the average droplet diameter decreased, whereas the oil mist concentration increased. As the cutting fluid flow increased, both of average droplet diameter and oil mist concentration increased.
\end{abstract}

Keywords: Metal cutting fluid; Oil mist; Diameter of droplets; Droplet distribution; Suspended microparticle

\section{Introduction}

The traditional flood lubrication consumes a large amount of cutting fluids. In the U.S., the annual consumption of cutting fluid is 100 million gallons, which is equivalent to 71 billion yuan. In Japan, the annual cost of cutting fluid is 42 billion yen. In 1994, the German processing industry consumed 75,491 tons of cutting fluids, of which 28,415 tons are watersoluble cutting fluid. The cost of cutting fluid accounts for $16 \%$ of total production costs. However, this number reaches $20-30 \%$ in the processing of some materials difficult to be processed. This is much higher than the cost of tools, which only account for $2-4 \%$ of total production costs. Costs of cutting fluid not only refer to the manufacturing costs but also include routine maintenance, pretreatment and handling costs. Although the cutting fluids are discharged after treatment, they are still harmful to the environment, especially to soil and water resources. Mineral oil is a major component of cutting fluid. Due to its extremely poor biodegradability, once it flows in soil or lakes, it is hard to be degraded and will stay for a long term. According to the U.S. Environmental Protection Agency, oil has acute lethal

\footnotetext{
${ }^{*}$ Corresponding Author
} 
toxicity to aquatic organisms as well as long-term sub-acute lethal toxicity. When the oil content exceeds $10 \mathrm{ppm}$ in water, it will lead to death of marine organisms. When the content exceeds 30ppm, it will lead to the death of freshwater fishes. Although the waste fluid is discharged after rigorous mineral oil recycling, its long-term accumulation in the water cannot be ignored. Additives in cutting fluids also generate environmental pollutions to various aspects. For example, extreme pressure agent is a pollutant in seawater. Meanwhile, phosphate in antirust agent leads to eutrophication of lakes, rivers and oceans, resulting in algae and red tides [1-4].

Using cutting fluid not only harms the environment, also harming the workers in plants. In the machining process, due to high pressure and high temperature, the vaporization and atomization of cutting fluids may happen and form mists of cutting fluids. The atomized microparticles of cutting fluid drift in the air and can be easily be inhaled by workers, leading to different types of lung diseases, including mild respiratory diseases, asthma and even cancers. The influence of cutting fluid aerosol on the health of workers might extend from lung diseases to risks of esophagus, stomach, pancreatic, prostate, colon and rectal cancers.

Cutting fluid generated from mechanical processing is mainly for timely transmission of abundant heat in the cutting area, lubrication of workpiece-cutter contact area, smear metal washing and corrosion prevention. With sufficient cooling and excellent lubrication effect, the traditional flood lubrication based on metal cutting fluid is highly appreciated in existing metal processing. It is estimated that the annual consumption of metal cutting fluid in the United States exceeds $3.8 \times 10^{8} \mathrm{~L}$ [5-11]. Metal cutting fluid will produce abundant oil mist particles in surrounding air. Many medical researches demonstrated that people exposed to these oil mist particles for long time are easier to have allergic dermatitis, malignant tumor and respiratory disease. Moreover, additive and pollutants in the cutting fluid may trigger acute asthma.

Occupational Safety and Health Administration of the United States stipulates $5 \mathrm{mg} / \mathrm{m}^{3}$ as the maximum mineral oil mist concentration. Later on, the Environmental Protection Agency of the United States became aware of great threats of small oil mist particles (e.g., longer lingering in air, higher chemical activity and stronger permeability) and shifted attentions from oil mist concentration to droplet size and proposed the concepts of PM10 and PM2.5. Particle diameter and size distribution are two important characteristics of oil mist.

General Motors (USA) carried out a thorough study on the oil mist problems generated from metal cutting fluid. They studied oil mist particles of different metal cutting fluids and found that non-water components of cutting fluid can increase the total oil mist in different processing [12-16]. They believed that this is caused by the quick evaporation of moisture content in oil mist while rest components still floating in the air. They also discovered a proportional relationship between oil mist particle size and concentration of the applied metal cutting fluid. However, they didn't study the influencing law of more parameters on the characteristics of oil mist through laboratory experiments.

In 2009, Broniarz et al., studied the oil mist produced from the emulsion pulverization of mineral oil emulsion, finding that the Sauter mean diameter of oil mist particles increased with the increasing of volume fraction of mineral oil in emulsion. In 1999, Kenneth L. Gunter and John W. Sutherland conducted an experimental survey on the effect of turning conditions on the oil mist concentration of metal cutting fluid. They explored the effects of rpm, workpiece diameter, nozzle diameter, cutting fluid concentration and sampling position on PM10 and PM2.5 systematically through experiments. Results demonstrated that rpm is the most important influencing factor of PM10 and the only one important influencing factor of PM2.5. No significant effect of cutting fluid concentration, nozzle diameter and vertical 
distance between nozzle and workpiece on the mass concentration of cutting fluid oil mist has been discovered [17-21].

\section{Categorization and Features of Suspended Air Particles}

American National Standards Institute (ANSI) defined Dust, Fumes and Mists in Industrial Health Engineering published in 1947. Hinds' Aerosol Technology defined the aerosol of other types, such as Smoke and Sprays. Table 1 describes the causes and typical size of aerosols [1-3].

Table 1. Causes and Size Range of Microparticles

\begin{tabular}{ccc}
\hline Name & Cause & Size Range $(\mu \mathrm{m})$ \\
\hline Dust & mechanical disruption & $>2$ \\
Fumes & condensation & $<1$ \\
Smoke & condensation & $<1$ \\
Mists & condensation or atomization & $5-1,000$ \\
Sprays & breakup of droplets & $>1^{2}$ \\
\hline
\end{tabular}

Commonly found aerosols in industrial environments are defined as follows:

Dusts refer to solid microparticles generated by grinding organic or inorganic substances, such as rocks, metals and grains. Dusts are hard to attach to other materials without static electric force. With the consideration of gravity and its influence, dusts are not prone to spread in the air. Fumes are solid microparticles formed by agglomeration of volatilized molten metal, which often present chemical reactions such as oxidation reaction. Microparticles in fumes usually form flocs. Mists are microparticles of suspended droplets formed by spraying, bubbling and splashing of liquids or formed in the agglomeration from gas to liquid. Smokes are aerosols due to incomplete combustion. Microparticles may be liquid or solid. Sprays are formed by the mechanical crushing of liquids. Homogeneous aerosol refers to the aerosol formed by the same chemical compositions. Monodisperse aerosol contains microparticles with basically the same size. Polydisperse aerosol contains microparticles with different sizes, which need to characterize sizes by statistical methods.

The microparticle size is essential to behavioral characteristics and toxicological characteristics of the aerosol. Microparticle size determines its kinematics in the air. Usually microparticle size refers to the diameter of particles and the aerodynamic diameter $d_{a}$ is used to describe the effective diameter of irregular (non-spherical) particles. $d_{a}$ is defined s follows: the diameter of a sphere with the unit density of $1 \mathrm{~g} / \mathrm{cm}^{3}$ moving with a low Reynolds number in the still air that has the same precipitation velocity of actual microparticles. The precipitation velocity refers to the velocity when the air buoyancy (resistance) of microparticles is equal to the gravity of microparticles. Table 2 shows the precipitation time of microparticles with different aerodynamic diameters.

\section{Table 2. The Relationship between Size of Microparticles in Aerodynamics and Precipitation Time}

\begin{tabular}{cc}
\hline microparticle size $\mathrm{d}_{\mathrm{a}}(\mu \mathrm{m})$ & precipitation time \\
\hline 0.5 & 41 hours \\
1 & 12 hours \\
3 & 1.5 hours \\
10 & 8.2 seconds \\
100 & 5.8 seconds \\
\hline
\end{tabular}


In some cases, Stokes diameter is used to define the sphere diameter that has the same density and precipitation velocity of actual microparticles. With the reduced microparticle size, the gravity was significantly reduced, when the dynamics behaviors of microparticles are mainly diffusion. In this case, the liquidity equivalent diameter is often used. The U.S. Environmental Protection Agency has classified the sizes, as shown in Table 3.

\section{Table 3. Categorization of Microparticle Size According to Aerodynamics}

\begin{tabular}{cc}
\hline Name & microparticles diameter in aerodynamics $\mathrm{d}_{\mathrm{a}}(\mu \mathrm{m})$ \\
\hline Super-large microparticles & $>10$ \\
Large microparticles & $2.5<\mathrm{d}_{\mathrm{a}}<10$ \\
Fine microparticles & $0.1<\mathrm{d}_{\mathrm{a}}<2.5$ \\
Super-fine microparticles & $<0.1$ \\
\hline
\end{tabular}

The component characteristics of suspended air microparticles in machining are diversified. The compositions of suspended microparticles are mainly decided by the compositions of raw materials. If suspended microparticles of aerosol formed in the processing have chemical reactions, such as oxidation reaction, the aerosol is known as secondary aerosol. Once the secondary aerosol is produced, the composition analysis will become complicated. Currently, devices to identify the composition of microparticles include $\mathrm{X}$-ray fluorescence spectrometry (XRF), proton-induced X-ray emission spectroscopy (PIXE), X-ray diffraction analyzer (XRD), optical emission spectrometer and mass spectrometer. Suspended particles sizes in machining also vary. Due to different microparticle sizes, its precipitation locations in human body also vary, imposing different degrees of influences on human health, as shown in Table 4. Therefore, to obtain specific information of the size, the sampling detection of aerosol and counting statistics were conducted. According to the statistics data, a columnar diagram to present the amount of microparticles and microparticle size can be produced. The same method can be used to describe the relationship between quality distribution and microparticle size. Distribution parameters of microparticle size can be further identified, like the mean, median and mode. Mean is obtained by the sum of microparticle sizes divided by the amount of microparticles, Medium is the value in the middle of microparticle sizes with ascending sort. Mode is the value most frequently appeared among all microparticle sizes. According to reports, inflammatory activities of low-toxicity particle are related with its large surface area, indicating that the microparticle size is the most influential factor for the toxicological response of microparticles, followed by chemical composition.

Table 4. The Relationship between Microparticle Size and its Sedimentary Location in Human Body

\begin{tabular}{cc}
\hline microparticle size $(\mu \mathrm{m})$ & sedimentary location in human body \\
\hline$>10$ & nasal cavity \\
$5-10$ & nasopharynx \\
$2-5$ & Mucociliary clearance in air tube and bronchia areas \\
$0.01-2$ & Lungs and pulmonary alveoli \\
$<0.01$ & expiratory clearance \\
\hline
\end{tabular}




\section{Impact of Suspended Air Particles on Health}

In the past few decades, according to the advantages and disadvantages of the coolant in machining, many studies have been conducted on coolant. Generally speaking, the goal to use coolant is for cooling, lubrication, corrosion and debris cleaning. The use of coolant can improve the service life of tools, the quality of the workpiece and effective cleaning of debris, thus greatly improving the processing conditions. However, substantial cooling liquid will produce considerable waste streams. To avoid the contamination of rivers, lakes and lands due to the use and disposal of coolant, the cooling pretreatment and processing is often needed, which will increase costs of cooling liquid waste. As a part of the coolant attaches on debris and the workpiece, it is inevitable to influence the environment, even for those rationally disposed cooling liquid waste. It is worth noting that costs of cooling pretreatment and processing are higher than its manufacturing costs, and treatment is not always effective. Thus, it is possible to cause water pollution.

Waste streams of the coolant will not only lead to environmental problems but also give rise to health and safety issues. According to the U.S. Bureau of Labor, in 2004, the employment rate of the manufacturing industry was less than $14 \%$, but causing a rate of nonfatal occupational diseases of $42 \%$. A part of these occupational diseases are lung diseases, which can be attributed to unacceptable operating ambient air quality. When concentrations of suspended microparticles in the operating ambient air exceed the standard, it will seriously endanger workers' respiratory health. According to related statistics, in 2000, due to the deposits of suspended air microparticles the respiratory system and the human body, leading to 2960 deaths. About 20-30\% adult asthma patients were caused by overproof suspended microparticles in the working environment. The fourth largest cause of death in the U.S. is chronic obstructive pulmonary disease. $15 \%$ of it is related with the overproof suspended microparticles in the working environment ${ }^{[12-17]}$. These data demonstrates that the harm of suspended microparticles in the working air of the manufacturing industry to workers' health.

The human respiratory system absorbs air from the atmosphere, and the air flows through the nose, throat, epiglottis, trachea, bronchi, and bronchium and enters the alveoli. Gas exchanges in the alveoli. In the gas exchange process, the oxygen enters the blood system and carbon dioxide into the alveoli. Through respiration, carbon dioxide is excreted. Suspended microparticles enter the human body and deposit in different locations of the respiratory system. The microparticle size is the major factor for the precipitation locations. According to different precipitation locations of microparticles in the respiratory system, suspended microparticles can be divided into three categories.

(1)Respirable part-all suspended air microparticles available in inhalation through the nose and mouth throat

(2)The part that enters thoracic cavity -all suspended air microparticles available in inhalation through throat

(3)The part that enters the respiratory region - suspended air microparticles available in inhalation and reaching the gas exchange region of lungs

Currently, sizes of suspended air microparticles of these three parts size can take $100 \mu \mathrm{m}$, $10 \mu \mathrm{m}$ and $4 \mu \mathrm{m}$ as sections respectively. We have noted that suspended air particles matters (PM) have different chemical compositions and sizes [1-4]. As shown in Table 1, suspended air microparticle size influences its penetration degree in the respiratory system and its penetration locations. Human lung function has evolved over thousands of years in the natural environment with suspended microparticles. Clearing mechanisms of suspended microparticles in bronchi and alveoli are useful to clear excessive residues of microparticles. However, overly exposure in an overproof environment of suspended microparticles will exceed the capability of clearing mechanisms of microparticles. When the human body 
inhales more suspended microparticles than the capability of clearing mechanisms, suspended microparticles will deposit in human body and produce the negative impact on biological effect of human body. The deposited microparticle will influence the first organ within its contact or other organs. Influences of suspended microparticles include allergies, irritation and carcinogenic effects, which may lead to pneumonia, respiratory diseases and lung tumors. The impact of suspended microparticles on health is subject to different factors, including the duration and frequency of exposure, workers' own body sensitivity and types of inhaled chemical substances of aerosol. In short-term or long-term exposure, symptoms may be observed. According to the toxicity of inhaled substances, short-term exposure may cause acute poisoning and long-term exposure or frequent exposure may lead to chronic poisoning. The influences of short-term exposure on health include irritation of nose, chest and eye, bronchitis, cough, edema, pneumonia, nausea and vomiting etc. Diseases from long-term exposure include asthma, emphysema, silicosis, lung cancer, laryngeal cancer, and urinary tract cancer and so on.

Since its foundation, the United States Environmental Protection Agency began to build related standards of the impact of suspended air microparticles on human health and the environment. In 1987, the Environmental Protection Agency established microparticle size standard, which required that the size of discharged microparticles should not be smaller than $10 \mu \mathrm{m}$. In 1997, the Agency presented a criterion, indicating that the size of discharged microparticles should not be smaller than $2.5 \mu \mathrm{m}$. Microparticles with the size smaller than $2.5 \mu \mathrm{m}$ can reach the gas exchange area of lungs through the respiratory system, even entering other organs, such as the olfactory nerve center. The China National Ministry of Environmental Protection announced on Jan.16, 2012 that PM2.5 and ozone (8-hour concentration) were included into the routine air quality assessment. The standard limit of PM10 and nitric oxide was also elevated. Some scholars verified that the indoor PM2.5 concentration of households was over 5 folds of PM2.5 concentration in the atmospheric environment. PM2.5 concentration of workshops was over 30 folds of that of households [37]. Working at such a concentration, workers are facing serious health problem.

Besides, cutting fluids are ideal environment for the growth of bacteria and fungi. Studies show that when $\mathrm{pH}$ of cutting fluid exceeds 10 , some extremophiles can still survive and grow in the extreme environments. Bacteria in cutting fluid will split the emulgator and reduce the lubricating ability of cutting fluids. They can also alter $\mathrm{pH}$ value of cutting fluid, which increases risks of corroding tool and workpiece. Moreover, cutting fluid with bacteria and fungi is risky for staff in the operating environment. In order to control the growth of bacteria in the cutting fluid, currently various types of fungicides, wetting agents, and sterilization agents are used in the industry. It is proved that even fungicides are used; some bacteria such as aeruginosa can still survive in the cutting fluid.

In order to control the growth of bacteria in the cutting fluid, chemical additives are often used in cutting fluid. Chemical additives are extremely harmful to the health of workers and the environment. If cutting fluid contains chemical additives, its own natural degradation capacity will be weakened. Some countries have banned the discharge of disposed wastes with fungicides in the sewage system. Spinosad and fungicides can maintain the function of cutting fluid but also put the health of workers in danger. Fungicides used in many factories will release methanol, a potential carcinogen [22-25]. According to International Agency for Research on Cancer, mineral oils used in metalworking are carcinogenic substances. With the exposure to the air full of suspended microparticles of mineral oils will lead to occupational skin cancer. Even those fungicides that do not release methanol are still dangerous to the human health and these fungicides are highly corrosive to the skin. 
In addition to fungicides, cutting fluid contains many other chemical substances, which are also very harmful to the environment and health. Extreme pressure (EP) cutting fluid are added with in-active sulfur and chlorine compound additives, which will react with the metal surface and form a protective film with low friction on the smooth surface. Although these chemical substances reduce the friction between tools and the workpiece surface, they are harmful to the health of workers and the environment. In the machining, chlorcosane in EP cutting fluid will produce Dioxin under the condition of heating and pressure. It is known to all that dioxin is a extremely harmful substance.

\section{Generation Mechanism of Oil Mist}

The circulation in pressure pump, intense impact with high-speed rotating workpiece or cutter and high-temperature working environment of metal cutting fluid determine the complicated generation mechanism of oil mist. Oil mist is the product of mechanical, physical and chemical effects. However, not all influencing factors have decisive effect on the generation of cutting fluid oil mist. Practically, pulverization mechanism and evaporation mechanism are two common generation mechanism of oil mist during metal cutting [2-5]. The pulverization mechanism of metal cutting fluid is equal to the conversion of mechanical energy into droplet surface energy. In this process, metal cutting fluid produce intensive impacts with cutter and high-speed rotating workpiece under certain pressure and flow rate, finally developing tiny particles floating in the surrounding air. Evaporation of metal cutting fluid mainly occurs in the cutting region. This is because considerable heats will be generated and transmitted to the cutting fluid during the processing, increasing the temperature of cutting fluid to its saturated temperature [26, 27]. At this moment, the solid-liquid interface of the cutting fluid will be boiling and produce vapor that will freezing with other small droplets or particles in surrounding air into oil mist. Figure 1 presents the generation mechanism of oil mist during metal grinding process.

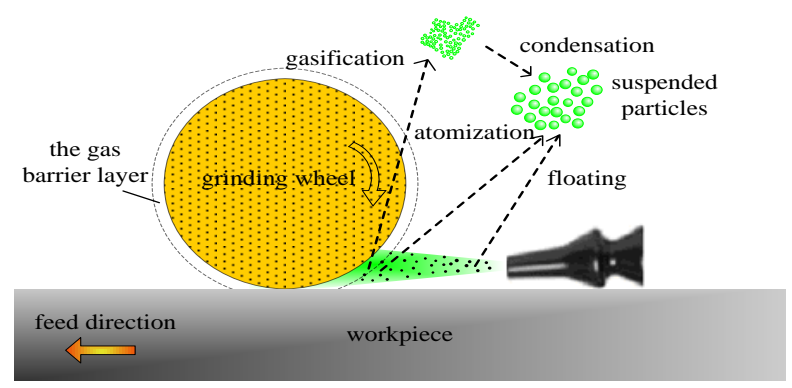

Figure 1. Generation of Suspended Microparticles in Grinding

When the cutting fluid is sprayed into the cutting area, it will intensely collide with the workpiece or tools in high-speed rotation, or evaporate at high temperatures after reaching the cutting area. These lead to the complex formation of ultimate suspended microparticles, during which mechanical, physical and chemical factors intertwine and act. In traditional flood lubrication, there are two mechanisms to form suspended microparticles. One is the high temperature in the cutting area that gasifies the cutting fluid, which is condensed and forms suspended microparticles in the air. The other is the intense collision between the cutting fluid and the workpiece or tools in high-speed rotation, leading to the atomization of cutting fluid into smaller suspended microparticles. Chen and others provided a formation model of suspended microparticles in traditional flood lubrication and used rotating disk for the circumference atomization of model. In the model, drop formation was divided into three 
stages, namely, liquid film formation stage, liquid strip formation stage and drop formation stage. Besides, they presented the computation equations of microparticle size after atomization.

\subsection{Types of Oil Mist}

There are three types of oil mist generated in the metal processing:

(1) Pure oil mist without solid powder generated from impacts with nozzle.

(2) Oil mist generated from high-temperature evaporation or firing during processing.

(3) Oil mist with grinding dust generated in the ground finish.

The droplet diameter range of oil mist differentiates due to its complicated generation mechanism and process. Generally speaking, oil mist generated from pulverization mainly exists in liquid droplet with a diameter range of $2-10 \mu \mathrm{m}$. Condensed oil vapor from evaporation of cutting fluid will produce tiny suspended solids $(<2 \mu \mathrm{m})$. The International Standard Organization (ISO) defined the behavior of different-size particles in respiratory tract: particles $(<100 \mu \mathrm{m})$ absorbed in the respiratory tract, particles $(<10 \mu \mathrm{m})$ subsided in lung and particles $(<4 \mu \mathrm{m})$ exhaled out.

\section{Oil Mist Control from Metal Cutting Fluid}

\subsection{Shield Close}

Currently, machinery mist reduction is preferred by most metal processing workshops to control the oil mist concentration, mainly including ventilator, oil mist catcher and machine shield [7,8]. Although these approaches are simple and effective, they still have some shortcomings. For example, ventilator only pumps oil mist in workshops to outdoor, but fails to solve the oil mist fundamentally. Oil mist catcher is inferior for its high maintenance and use cost as well as decreasing efficiency with the increase of oil mist catching. Machine shield has many application limitations and complete machine shield is impossible in most cases.

\subsection{Chemical Control}

Chemical control of cutting fluid oil mist is based on the reasonable selection of metal cutting fluid and application of additive in cutting fluid. To satisfy different processing technical demands, there are various classes of cutting fluid available. Compared with waterbased cutting fluid, oil-based cutting fluid has better lubricity, stronger bacterial resistance and simpler recycle, but poorer cooling capacity, higher oil mist production and heavier environmental pollution. As a result, oil-based metal cutting fluid produces less oil mist compared to the oil-based metal cutting fluid in practical metal processing.

It has been confirmed an efficient oil mist control to add macromolecular polymer in metal cutting fluid. This is because macromolecular polymer can facilitate the quick settlement of small oil mist particles. However, polymer additive claims higher cost of cutting fluid and regular replenishment due to its restricted molecular decomposition during the recycle.

\subsection{Motion Condensation}

With the enhancement of environmental awareness and development of manufacturing technologies, some new efficient techniques and methods for reducing cutting fluid oil mist have been developed recently. Motion condensation is one representative method. Motion condensation, based on the motion condensation mechanism, uses a set of exclusive spraying 
system to produce large droplets absorb and subside oil mist particles floating in the air, thus shortening the floating time of oil mist in air effectively. Experimental results have confirmed its efficiency in reducing oil mist concentration in working environment. Additionally, many other environmental-friendly processing techniques with less cutting fluid oil mist have been developed, such as dry cutting, minimum quantity lubrication, oil mist lubrication, etc., However, all of them are facing with some practical application limitations.

\section{Theoretical Model}

Oil mist generated from metal cutting fluid has attracted wide attentions for its great threats to human beings and natural environment. To explore the effect law of processing conditions on the generation of cutting fluid oil mist and propose the optimal effective oil mist control, many attempts have been made by scientists to control the oil mist production, mainly including theoretical analysis modeling and experimental verification.

Among so many theoretical model concerning the effect law of processing conditions on the cutting fluid oil mist, Erika Sujova proposed his theoretical model. He believed that oil mist is mainly produced by the high-temperature evaporation of cutting fluid in the cutting region, liquid aerosol generated in surrounding environment, rotation of workpiece or cutter, and splashed cutting fluid upon impacts with workpiece or cutter under certain pressure. During metal processing, a layer of liquid film will be produced on the workpiece surface by the splashed cutting fluid. The initial disturbance on this liquid film will develop an imbalanced counterforce. The surface tension of the liquid film will offset this counterforce and tries to restore the balance. However, aerodynamic force or centrifugal force will increase the disturbance on the liquid film and break the balance. When this relative motion intensifies, a pressure distribution will be developed by the air to increase the disturbance amplitude. When the disturbance amplitude increased to the threshold, the surface tension of the liquid film is inadequate to maintain the integrity of the liquid film. Consequently, the liquid film will be broken into droplets and produce oil mist. The model of oil mist generation from spraying impact of cutting fluid on the rotating workpiece was proposed according to mathematical properties. When cutting fluid sprays to the rotating workpiece (radius: R) at the angular velocity of $\omega$ on the horizontal plane (Figure 2), three spraying patterns will be observed due to the different cutting fluid flow, namely, droplet, liquid belt and liquid film.

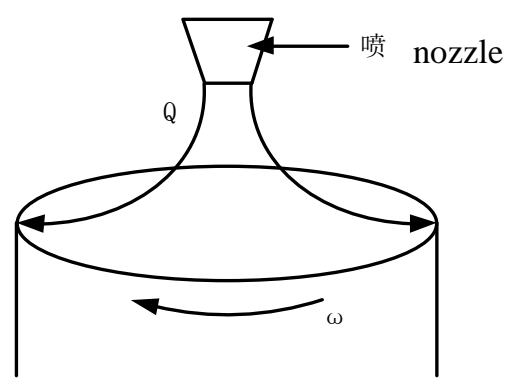

Figure 2. Cutting Fluid Spraying Onto Rotating Workpiece

\subsection{Droplet Generation Mechanism}

A layer of thin liquid film will be covered on the workpiece surface under extremely small cutting fluid flow $(\mathrm{Q})$. When the centrifugal force exceeds the surface tension $(\gamma)$, droplets 
will be sprayed form the surface edge. The force balance at the separation of droplets and the surface edge is:

$$
\frac{\pi D^{3}}{6} \rho R \omega^{2}=\pi D \gamma
$$

Where $\rho$ is the fluid density.

The droplet diameter (D) can be calculated from:

$$
D=\frac{c}{\omega}\left(\frac{\gamma}{\rho \cdot R}\right)^{\frac{1}{2}}
$$

Theoretically, $c=\sqrt{6}$, but practically it is impossible because the centrifugal force exceeds the surface tension at the formation of droplets. In this process, the distribution of $D$ is determined by fluctuation amplitude. In Figutr 3, a lot of small droplets are separated from the large droplet edge due to the inertia force.

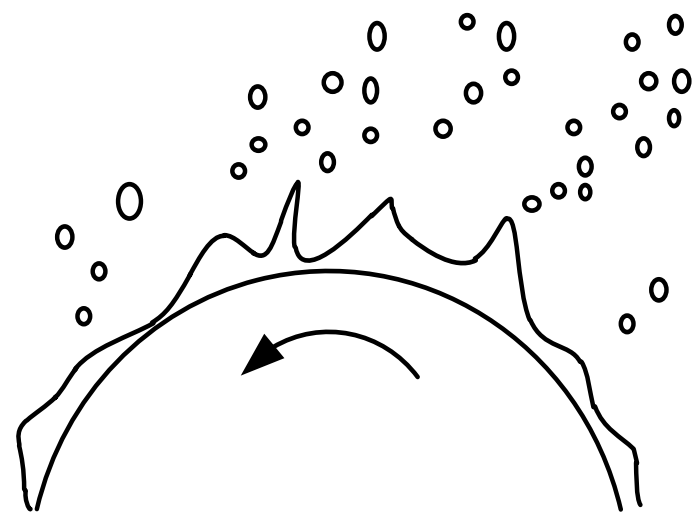

Figure 3. Droplet Generation

\subsection{Liquid Belt Generation Mechanism}

The liquid film begins to present waving disturbance after $\mathrm{Q}$ increased to the specified value. Many unstable liquid belts will be produced surrounding the liquid film and broken into big and small droplets (Figure 4). The outward extension trace of liquid belts at the liquid film edge can be expressed approximately by:

$$
\begin{aligned}
& x=R \cos (\omega t)+R \omega t \sin (\omega t) \\
& y=R \sin (\omega t)-R \omega t \cos (\omega t)
\end{aligned}
$$

According to experience, the diameter of liquid belt (d1) can be defined:

$$
d_{l}=c^{\prime} R\left\{\left(\frac{1}{N_{l}}\right)^{\frac{2}{7}}\left(\frac{\gamma}{\rho R^{3} \omega^{2}}\right)^{\frac{2}{7}}\left(\frac{\rho Q^{2}}{R^{3} \gamma}\right)^{\frac{1}{7}}\right\}
$$

Where $\mathrm{N}_{1}$ is the amount of liquid belts and $c^{\prime}$ is an empirical constant. 


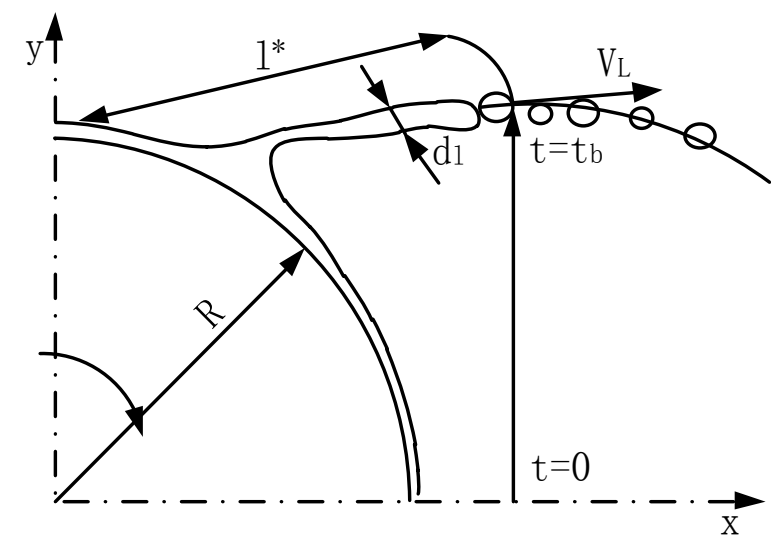

Figure 4. Liquid Belt Generation

Based on Weber's theory, k calculated the approximate D:

$D=\left(\frac{3 \pi}{\sqrt{2}}\right)^{\frac{1}{3}} d_{1}\left|1+\frac{3 \mu}{\left(\rho \gamma d_{1}\right)^{\frac{1}{2}}}\right|^{\frac{1}{6}}$

D can be calculated by substituting (5) into (6). Compare it the empirical formula (7) of D:

$D=5.75 N^{-0.79} Q^{0.32} R^{-0.69} \rho^{-0.29} \gamma^{0.26}(1+0.23 \mu)^{0.65}$

Where $\mathrm{N}$ is rpm and $\mu$ is dynamic viscosity.

\subsection{Liquid Film Generation Mechanism}

High flow and rpm will cause intensive fluctuation of surrounding medium and break the liquid film directly into small droplets. During the processing, oil mist is generated by the above three spraying patters simultaneously at different regions rather than the consequence of a single spraying pattern (Figure 5).

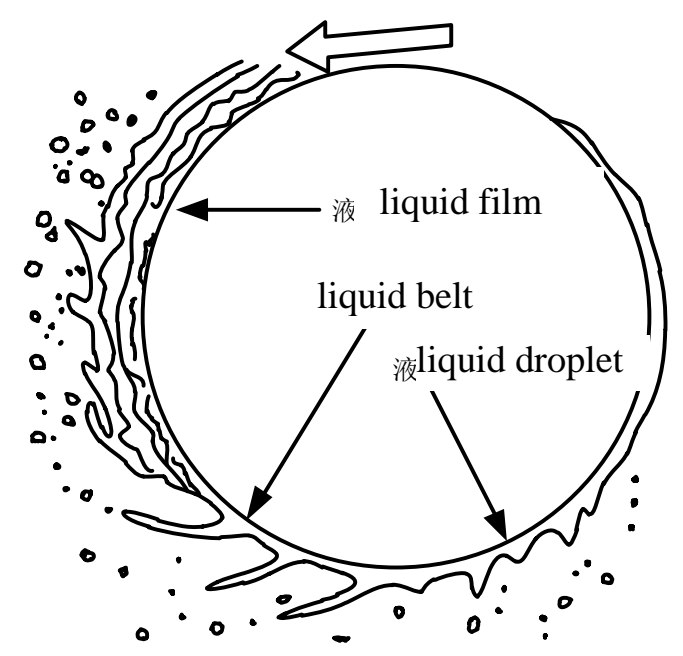

Figure 5. Generation of Three Types of Oil Mist 


\section{Experimental Study}

\subsection{Experimental Setup}

In this experiment, a droplet diameter measuring system was equipped in the dense region of oil mist, which enables to observe the droplet distribution in air and scan the morphology of a single droplet accurately through its confocal microscopy. Next, droplet distribution and diameter were calculated through computer. The lathe was sealed up and the nozzle of cutting fluid was fixed vertically about $1 \mathrm{~cm}$ above the rotating workpiece to reduce droplets (Figure $6)$.

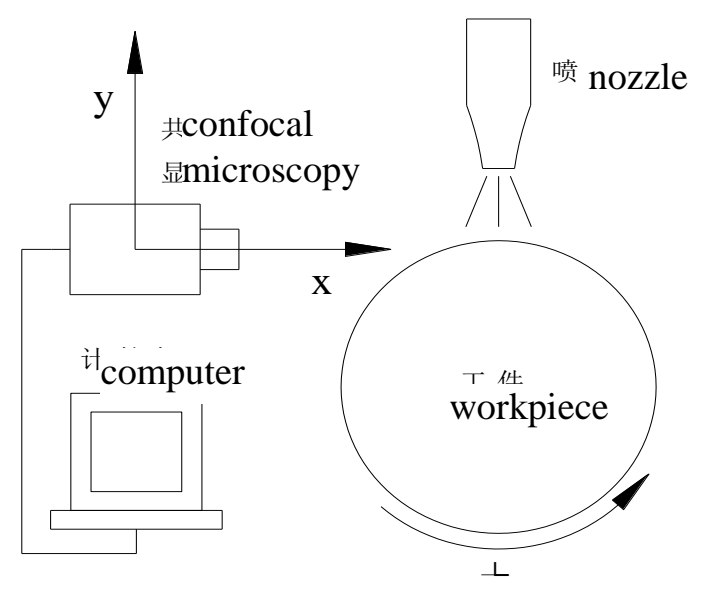

Figure 6. Experimental Apparatus

\subsection{Droplet Diameter Distribution}

The theoretical and experimental results of droplet diameter distribution were shown in Figure 7. Theoretically, droplet diameter is subject to lognormal distribution, mainly ranging between $0.2 \sim 5 \mu \mathrm{m}$. Practical droplet diameter also is subject to lognormal distribution according to the experimental results, mainly ranging between $0.5 \sim 5 \mu \mathrm{m}$. This indicates that the theoretical curve of droplet diameter basically agrees with the experimental results. Therefore, it can determine that oil mist size generated from cutting fluid is subject to the lognormal distribution.

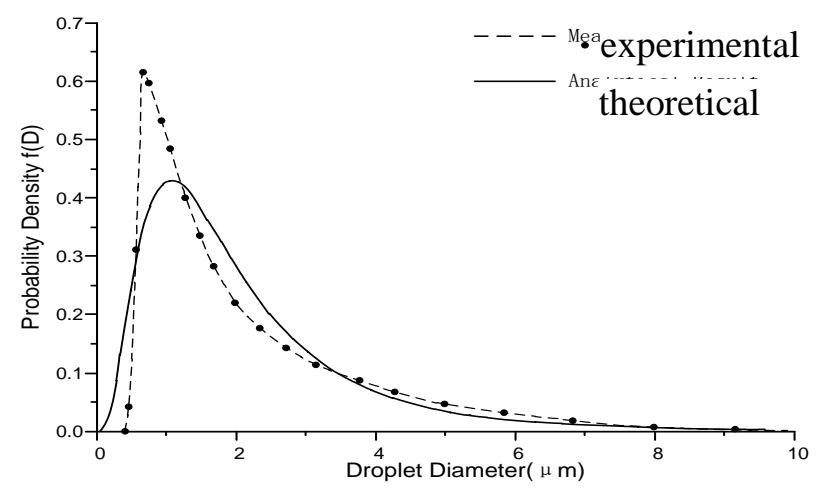

Figure 7. Comparison of Experimental and Theoretical Droplet Diameter Distribution 


\subsection{Effects of Cutting Fluid Flow on the Concentration and Mean Diameter of Droplets}

Although the generation mechanism of oil mist from metal cutting fluid is very complicated and involves various influencing factors, not all influencing factors are decisive to the generation of oil mist. Abundant experiments demonstrated that cutting fluid oil mist is mainly influenced by rpm and cutting fluid flow. To explore the effect law of cutting fluid flow and rpm on the concentration and mean diameter of cutting fluid oil mist, this experiment involved two cutting fluid flows $(0.5 \mathrm{~L} / \mathrm{min}$ and $0.7 \mathrm{~L} / \mathrm{min})$ and five $\mathrm{rpm}(1000$, $1500,2000,2500$ and 3000). Based on a rotating workpiece with $60 \mathrm{~mm}$ diameter, the experiment observed the concentration and Sauter mean diameter (SMD) of oil mist for 30s to discuss. The effects of cutting fluid flow and rpm on the concentration and SMD of oil mist were presented in Figure 8 and Figure 9.

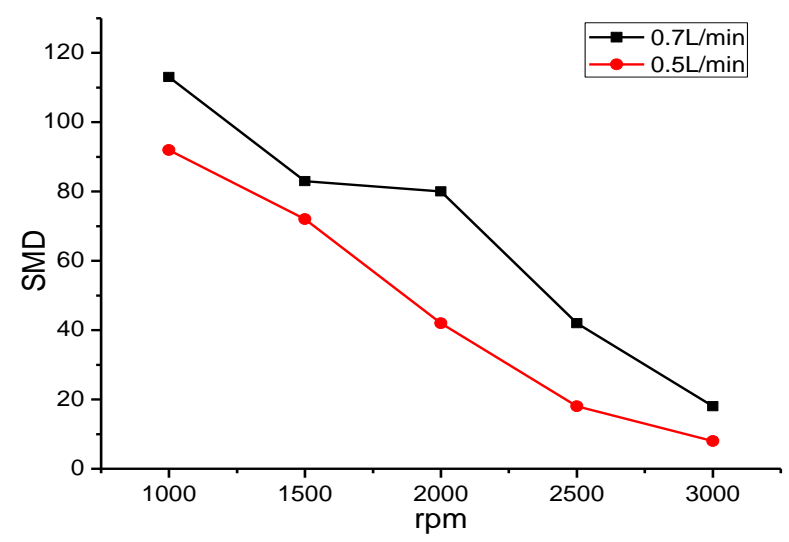

Figure 8. Effects of Cutting Fluid Flow and rpm on SMD of Droplets

In Figure 8, the effects of cutting fluid flow $(0.5 \mathrm{~L} / \mathrm{min}$ and $0.7 \mathrm{~L} / \mathrm{min})$ and $\mathrm{rpm}(1000,1500$, 2000, 2500 and 3000) on SMD of droplets were tested under same of other processing conditions. Results demonstrated larger SMD of droplets under $0.7 \mathrm{~L} / \mathrm{min}$ compared to $0.5 \mathrm{~L} / \mathrm{min}$ at all five rpm. In Figure 9, the concentration of droplets under $0.7 \mathrm{~L} / \mathrm{min}$ is higher compared to $0.5 \mathrm{~L} / \mathrm{min}$ at all five $\mathrm{rpm}$. To sum up, given same processing conditions, the concentration and SMD of droplets are significantly proportional to cutting fluid flow.

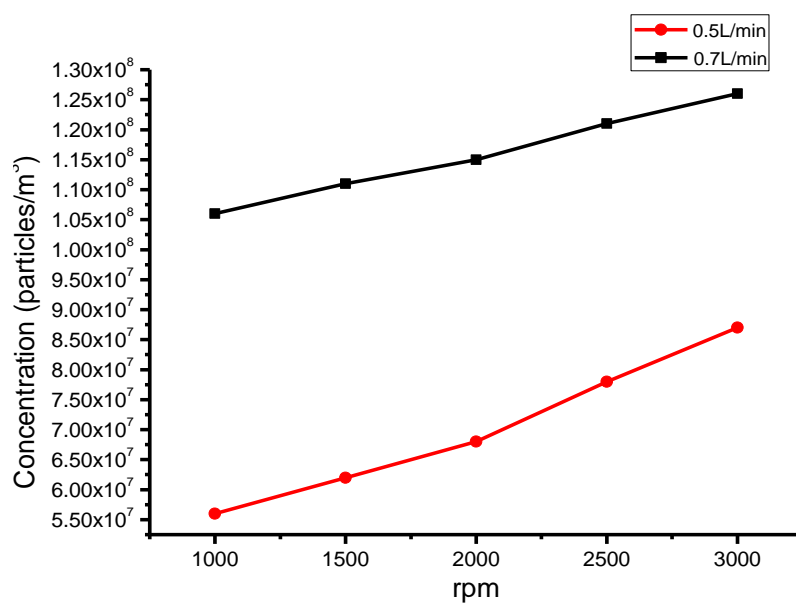

Figure 9. Effects of Cutting Fluid Flow and rpm on Concentration of Droplets 


\subsection{Effect of Rpm on the Concentration and Mean Diameter of Droplets}

In Figure 8, given same processing conditions, SMD of droplets under both $0.7 \mathrm{~L} / \mathrm{min}$ and $0.5 \mathrm{~L} / \mathrm{min}$ decreases with the increase of rpm from 1000 to 3000 , decreasing from $112 \mu \mathrm{m}$ to $18 \mu \mathrm{m}$ and from $91 \mu \mathrm{m}$ to $9 \mu \mathrm{m}$, respectively. In Figure 9, given same processing conditions, concentration of droplets under both $0.5 \mathrm{~L} / \mathrm{min}$ and $0.7 \mathrm{~L} / \mathrm{min}$ increases with the increase of $\mathrm{rpm}$ from 1000 to 3000 , increasing from $5.6 \times 10^{7} / \mathrm{m}^{3}$ to $8.7 \times 10^{7} / \mathrm{m}^{3}$ and from $1.07 \times 10^{8} / \mathrm{m}^{3}$ to $12.7 \times 10^{8} / \mathrm{m}^{3}$, respectively. This reveals the negative correlation between rpm and SMD of droplets, but positive correlation between rpm and concentration of droplets.

\section{Conclusion}

Equation (7) also demonstrates that D is inversely proportional to $\mathrm{N}$, but proportional to $\mathrm{Q}$. In other words, higher rpm results in smaller diameter of oil mist and higher cutting fluid flow contributes larger diameter of oil mist. This theory was confirmed by experimental results.

Viewed from the generation mechanism of cutting fluid oil mist, increasing rpm exerts stronger impact force on the cutting fluid, which intensifies the pulverization of cutting fluid. Meanwhile, increasing heats in the cutting zone intensifies the evaporation of cutting fluid. They finally increase the cutting fluid oil mist to certain extent.

Both theoretical and experimental results confirmed the significant effects of cutting fluid flow and rpm on the concentration and mean diameter of cutting fluid oil mist. Higher cutting fluid flow has higher concentration and SMD of droplets. Higher rpm has smaller SMD but higher concentration of droplets.

\section{Acknowledgements}

This research was financially supported by the National Natural Science Foundation of China (50875138; 51175276), the Shandong Provincial Natural Science Foundation of China (Z2008F11; ZR2009FZ007).

\section{Conflict of Interests}

The authors hereby confirm that no conflict of interest exists for this article.

\section{References}

[1] Shokrani, V. Dhokia, S.T. Newman, "Environmentally conscious machining of difficult-to-machine materials with regard to cutting fluids", International Journal of Machine Tools \& Manufacture, vol. 57, (2012), pp.83-101

[2] E. Sujova, "Contamination of the working air via metalworking fluids aerosols", Engineering Review, vol.32, no.1, (2012), pp. 9-15.

[3] K. Donaldson, X.Y. Li, and W. MacNee, "Ultrafine (nanometer) Particle Mediated Lung Injury", Journal of Aerosol Science. vol.29, no.5, (1998), pp. 553-560

[4] D. J. Michalek, W.W.S Hii, J. Sun, et al, "Experimental and analytical efforts to characterize cutting fluid mist formation and behavior in machining:, Applied occupational and environmental hygiene, vol18, no.11, (2003), pp.842-854

[5] Y. Yue, J. Sun, K. L. Gunter, et al, "Character and behavior of mist generated by application of cutting fluid to a rotating cylindrical workpiece, Part 1: Model development", Journal of manufacturing science and engineering, vol.126, no.3, (2004), pp. 417-425

[6] J. Sun, C. Ju, Yue Y, et al, "Character and behavior of mist generated by application of cutting fluid to a rotating cylindrical workpiece, Part 2: Experimental validation”, Journal of manufacturing science and engineering, vol.126, no.3, (2004), pp. 426-434

[7] D. P. Adler, W. W. S. Hii, Michalek D J, et al, "Examining the role of cutting fluids in machining and efforts to address associated environmental/health concerns", Machining Science and technology, Vol.10, no.1, (2006), pp.23-58. 
[8] T J Ko, S H Park, H S Kim, "Experimental verification of the mist generation mechanism in turning", International Journal of Machine Tools and Manufacture, vol.43, no.2, (2003), pp.115-120.

[9] C. H. Li, G. Q. Cai and S. C Xiu, "Auto-correlation study on the surface profile finished by abrasive jet with grinding wheel as restraint”, Int. J. Computer Applications in Technology, Vol.29, nos.(2-4), (2007), pp.262-265

[10] C. H. Li, Y. L Hou, C. Du and, Y. C. Ding, "An analysis of the electric spindle's dynamic characteristics of high speed grinder", Journal of Advanced Manufacturing Systems, Vol.10, no. 1, (2011), pp.159-166.

[11] K L Gunter, J W Sutherland, "An experimental investigation into the effect of process conditions on the mass concentration of cutting fluid mist in turning", Journal of Cleaner Production, Vol. 7, no.5, (1999), pp. 341350.

[12] L Qian, J Lin, H Xiong, et al, "Theoretical investigation of the influence of liquid physical properties on effervescent atomization performance", Journal of Fluids Engineering, Vol.133, no.10, (2011), pp.101-115.

[13] Z Chen, K Wong, W Li, et al, "Cutting fluid aerosol generation due to spin-off in turning operation: analysis for environmentally conscious machining", Journal of manufacturing science and engineering, Vol.123, no.3, (2001), pp.506-512.

[14] C. Li H, Y. L. Hou, Ding Y. C., Cai G. Q, "Feasibility investigations on compound process: a novel fabrication method for finishing with grinding wheel as restraint", International Journal of Computational Materials Science and Surface Engineering, Vol.4, no.1, (2011), pp. 55 - 68.

[15] .H. Li, S. Wang, Q. Zhang and Y.C. Ding, "Influence of Unbalanced Response of Ultra-high Speed Grinder Spindle on Dynamic Performance”, Int. J. Materials and Product Technology, Vol. 45, nos. (1/2/3/4), (2012), pp.119-131.

[16] P Adler, W W S Hii, D J Michalek, et al, "Examining the role of cutting fluids in machining and efforts to address associated environmental/health concerns", Machining Science and technology, Vol.10, no.1, (2006), pp.23-58.

[17] T J Ko, S H Park, H S Kim, "Experimental verification of the mist generation mechanism in turning", International Journal of Machine Tools and Manufacture, Vol.43, no.2, (2003), pp.115-120.

[18] D S Sokolović, W Höflinger, Sokolović R M Šečerov, et al, "Experimental study of mist generated from metalworking fluids emulsions", Journal of Aerosol Science, Vol.61, no.2, (2013), pp. 70-80.

[19] . H. Li, Z. R. Liu, Hou Y. L., et al., "Critical conditions for brittle-ductile removal transition in nano-ZrO2 dental ceramic grinding", International Journal of Machining and Machinability of Materials, vol. 11, no. 4, (2012), pp. 342-352.

[20] Dasch J M, D'arcy J B, Kinare S S, et al, "Mist generation from high-speed grinding with straight oils", Tribology Transactions, Vol.51, no.3, (2008), pp.381-388.

[21] Li C. H., Han Z. L., Zhang Q., et al., "Modeling and Experimental Investigation of Pressure Field in the Grinding Zone with Nanoparticle Jet of MQL", Advances in Mechanical Engineering, vol. 2013, (2013).

[22] Y. L. Hou, C. H. Li, Z. L. Han, J.Y. Li and Y. C. Ding, "Examination of the Material Removal Mechanisms During the Abrasive Jet Finishing of 45 Steel", Advanced Science Letter, Vol. 4, nos.(4-5), (2011), pp.14781484.

[23] S Wang, C H Li, Y C Ding. "Investigation into Speed and Temperature Field of Metal Drop in High-melting Metal Arc Spraying”, International Journal of Control and Automation, Vol.5, no.3, (2012), pp.237-248.

[24] C.H Li., J. Y. Li, S. Wang, I., "Modeling and numerical simulation of the grinding temperature field with nanoparticle jet of MQL", Advances in Mechanical Engineering, vol. 2013, (2013).

[25] J. Dongzhou, C. Li, D. Zhang, S. Wang and Y. Hou, "Investigation into the Formation Mechanism and Distribution Characteristics of Suspended Microparticles in MQL Grinding", Recent Patents on Mechanical Engineering ,Vol.7, no.1, (2014), Pp. 52-62

[26] J. Dongzhou, C. Li and L. Runze, "Modeling and Experimental Investigation of the Flow Velocity Field in the Grinding Zone, International Journal of Control and Automation,vol.7, no.2, (2014), pp.405-416.

[27] Z. L. Han and C. H. Li. "Theoretical Modeling and Simulation of Airflow Field near Grinding Wheel", International Journal of Control and Automation, vol.6, no.4, (2013), pp.145-155 
International Journal of Control and Automation Vol.7, No.7 (2014) 